\title{
Linx
}

Revue des linguistes de l'université Paris X Nanterre

38 | 1998

L'acquisition de la temporalité en situation bilingue

\section{Les ponts interlangues}

Language links

\section{Marlène Dolitsky}

\section{OpenEdition}

Journals

Édition électronique

URL : http://journals.openedition.org/linx/865

DOI : $10.4000 /$ linx.865

ISSN : 2118-9692

\section{Éditeur}

Presses universitaires de Paris Nanterre

\section{Édition imprimée}

Date de publication : 1 décembre 1998

Pagination : 59-64

ISSN : 0246-8743

Référence électronique

Marlène Dolitsky, "Les ponts interlangues », Linx [En ligne], 38 | 1998, mis en ligne le 26 juin 2012 consulté le 01 mai 2019. URL : http://journals.openedition.org/linx/865; DOI : 10.4000/linx.865

Ce document a été généré automatiquement le 1 mai 2019.

Département de Sciences du langage, Université Paris Ouest 


\title{
Les ponts interlangues
}

\author{
Language links
}

\author{
Marlène Dolitsky
}

\section{Introduction théorique}

1 Une controverse qui a longtemps traversé le études du bilinguisme, c'est la question de savoir si l'individu bilingue dispose d'un système de représentation ou de deux. Les premières discussions sur ce sujet ont leur source chez Weinreich (1974) dans sa théorie des types de bilinguisme : composé, coordonné ou subordonné, et dans les expériences de Lambert (1972) fondées sur cette théorie. Cependant, une telle catégorisation globale du bilinguisme est aujourd'hui écartée, bien que la question reste encore posée.

Quant à l'acquisition initiale bilingue du langage,Volterra et Taeschner (1977) considèrent qu'il n'y a qu'un seul système linguistique à l'étape initiale. L'enfant passerait ensuite par deux autres étapes avant d'arriver à "un vrai bilinguisme adulten": un stade de différenciation lexicale sans différenciation syntaxique, puis un stade de différenciation lexicale et syntaxique où l'enfant ne choisit pas lui-même le système dans lequel il puise pour produire un énoncé à un moment donné, mais où la production serait déclenchée par l'interlocuteur de l'enfant.

3 Cependant, cette perspective sur l'acquisition du bilinguisme est fortement réfutée par des travaux récents. De Houwer (1995), après avoir présenté les arguments de la controverse, conclut que "from very early on, the bilingual child makes contextually sensitive linguistic choices that draw on a developing knowledge of two separate language systems" (p. 248). Les travaux de Quay (1998) montrent que dès l'âge de 1,7 à 1,8, l'enfant fait preuve d'une capacité à choisir les éléments linguistiques correspondant à la langue de l'interlocuteur et/ou du contexte, sans pour autant que ceux-ci déclenchent automatiquement l'utilisation d'un système ou de l'autre.

4 On a rejeté depuis longtemps l'hypothèse que l'enfant acquiert la langue par simple imitation des adultes qui l'entourent et par un conditionnement skinnérien. On considère que l'enfant écoute, découpe et analyse le langage qu'il entend et qu'il élabore une 
structure qui est la base de ses propres productions. Sa restructuration du langage l'amène à une sur-généralisation des règles du langage adulte, avec des erreurs typiques du genre *j'ai perdé, *j'ai allé ou en anglais : *I doed, *I goed, ${ }^{*}$ I brang.

5 On pourrait supposer que le même genre de surgénéralisation se produit chez l'enfant bilingue entre les deux langues, surtout lorsque les langues offrent des ressemblances.

\section{Le sujet : Dexter}

6 L'hypothèse que nous proposons dans ce qui suit se fonde sur l'étude d'un enfant bilingue, français/anglais, qui s'appelle Dexter. Il est né en France de père français et de mère américaine et linguiste. Il vit avec sa mère qui ne lui parle qu'en anglais. Toute autre personne dans son entourage en France lui parle en français; toute personne dans son entourage aux USA où il va en vacances lui parle en anglais. Il est scolarisé dans une école maternelle publique française.

7 En suivant l'acquisition du bilinguisme de Dexter, ce qui se faisait remarquer c'est que les erreurs "persistantes" avaient lieu surtout là où les structures de ses deux langues se ressemblent. L'exemple le plus frappant est son utilisation du morphème RE-.

\section{Le morphème "RE-"}

8 Ce qui nous intéresse ici, c'est l'utilisation par l'enfant du morphème RE-. En français ainsi qu'en anglais, le préfixe RE-, qui se combine avec des lexèmes verbaux, est un formant de dérivation lexicale à valeur temporelle :

9 1) soit il marque une action qui permet un retour au point de départ d'une situation extralinguistique : il présuppose à la fois un état du monde et un changement de cet état ; il pose un retour à l'état d'origine ;

2) soit il marque une deuxième occurrence, une itération de l'action.

11 Un synonyme en anglais pour le premier serait "back", et pour le second "again". Cependant ce formant est soumis à une contrainte locale d'application de la règle d'affixation, qui ne lui permet pas d'entrer en combinaison avec l'ensemble de la catégorie des verbes - de fait, la règle est très sélective. Son utilisation dans les deux langues diffère au niveau des contraintes locales, c'est-à-dire au niveau des lexèmes verbaux avec lesquels ce morphème peut normalement se lier. Des exemples en anglais sont : REtie, REdo, REmake. En français on trouve : REnouer, REfaire, REvenir, REmettre.

12 On a remarqué une généralisation persistante de ce morphème chez Dexter (âge : 4,0 à 4,9), dans des phrases telles que :

* I REask him* I REcome home* I REfind the black fish *I REput it on the table

*I REcaught it like this * We REgo to Tommy's house.

13 Ces exemples montrent une compétence dérivationnelle bien acquise, mais représentent des constructions socialement illégitimes puisque ces verbes ne figurent pas sous cette forme dérivée dans le lexique attesté (Corbin 1987 : 71) de l'anglais. Il est important de 
remarquer que le français permet une dérivation avec RE- pour les analogues de ces verbes: REdemander, REvenir, REtrouver, REmettre, à l'exception du dernier : “Raller" et, pour attraper, une suppression de (e) du RE- pour former Rattrapper.

\section{Discussion}

Il y a plusieurs façons d'interpréter l'utilisation que Dexter fait de RE- en anglais. Volterra et Taeschner (1978) suggéreraient que cette utilisation signifie que l'enfant n'a qu'un système syntaxique, et la surgénéralisation de l'occurrence de RE à travers les deux langues confirmerait leur hypothèse. En se référant aux travaux de Grosjean (1982), par contre, l'utilisation inappropriée de RE- en anglais serait considérée comme un transfert de la langue dominante à la langue subordonnée. Par définition il y aurait donc l'hypothèse de deux systèmes séparés, puisqu'il ne s'agit pas d'identité mais de transfert.

15 Au contraire de Haugen (1956), selon qui un adulte, élevé dans un milieu bilingue depuis son enfance, a une compétence linguistique plus vaste que celle d'un monolingue mais plus étroite que celle de deux monolingues, Hoffman (1991:79) affirme que la compétence bilingue n'est pas la somme des compétences de deux parties séparées (un code et l'autre), mais qu'elle comprend aussi une compétence qui relie les éléments des deux codes. L'adulte bilingue aurait donc une compétence linguistique plus vaste que celle de deux monolingues.

16 En accord avec Hoffman, nous pensons que l'enfant bilingue, qui analyse et restructure les deux langues de son environnement, "remarque" des ressemblances entre les deux langues. Dans le cas de RE-, l'enfant remarquerait son existence dans les deux langues ainsi que la similitude sémantique et de maniement syntaxique. Il les marquerait donc comme semblables. Au lieu de voir cette extension comme un transfert d'une langue à l'autre, ou comme une preuve que l'enfant n'a qu'une langue, on préfère interpréter cette utilisation comme le signe d'une tentative de l'enfant de structurer l'ensemble de son environnement linguistique, où il développerait les règles de dérivation et de leurs contraintes pour chaque langue séparément, tout en cherchant de quelle manière les deux se ressemblent et se différencient. Ce qu'il reconnait comme "semblable" servirait en quelque sorte de "pont". Ces ponts serviraient comme base de règles qui relient les deux langues et seraient sous la forme de morphèmes marqués comme "semblables" (Klausen et. al. 1992).

17 On peut donner comme autre exemple de pont linguistique l'utilisation par Dexter de la préposition $O F$ :

They took the crown of a galette. A galette crown. They took a galette crown. $(4,6)$

It was the mouse that was the friend of Dumbo. In the cassette of Dumbo $(4,6)$

What's the name of your dog? I have a kitty. The name of mykitty is Kitty. $(4,8)$

18 On se rend compte que Dexter a identifié les similitudes de structure entre les morphèmes $O F$ et $D E$ dans les constructions du génitif et du complément de nom. Mais il y a une différence liée aux contextes d'emploi : en français l'utilisation de DE est obligatoire pour ces types de constructions, alors qu'en anglais of ne s'emploie qu'avec des 
syntagmes nominaux longs. L'utilisation de $O F$ dans ces exemples est une surextension de son usage à des syntagmes nominaux courts. Il est à remarquer (et cette observation est valable pour les deux cas) que la surextension de la règle peut être considérée d'un point de vue interlinguistique ou intralinguistique: ou bien l'enfant n'a pas perdu les limitations d'usage en anglais, ou bien il a exporté le modèle du français vers l'anglais. En l'absence de preuve à l'appui de l'une ou l'autre version, nous avançons l'hypothèse que la règle en français a eu une influence sur la formation de la règle en anglais, sans qu'il y ait pour autant transfert ou interférence à proprement parler.

D'autres confirmations de l'hypothèse de ponts qui relieraient les langues peuvent être trouvées dans les expériences qui utilisent des techniques d'amorçage sémantique. Beauvillain et. al. (1989:58) présentent des expériences (les leurs et celles d'autres auteurs) où l'on a observé un effet de facilitation sur des équivalents de traduction reliés morphologiquement, et ils en concluent que "sans qu'il soit nécessaire de faire appel à l'existence d'une représentation commune, il est possible de considérer l'information morphologique comme représentée dans le lexique sous forme de relations privilégiées". D'après ce que l'on a observé de l'utilisation de RE- chez Dexter, ces relations privilégiées, ou "ponts" peuvent exister entre des morphèmes permettant des dérivations, ce qui pourrait faire l'objet d'études ultérieures.

\section{Morphologie}

Hoffman (1991:69) a suggéré que les personnes bilingues pourraient avoir un sens plus aigu que les monolingues de la structuration morphologique. La création par l'enfant de combinaisons socialement illégitimes de racines et d'affixes - comme celles citées précédemment à propos de l'utilisation de RE - confirme l'hypothèse de l'utilisation d'un lexique décomposé en morphèmes (Badecker et Caramazza 1991).

Dans le prolongement de cette hypothèse, on peut avancer que l'utilisation des constructions dans les deux langues (tout en étant illégitimes dans l'une des deux) suggère que les deux langues ont été analysées de manière morphologique. L'enfant a donc trouvé pour ce morphème une équivalence tant pour la forme phonologique que pour le sens.

Cela voudrait dire que les enfants sont conscients du fait qu'ils reçoivent des informations des deux langues, mais qu'ils essayent activement de jeter des ponts entre elles.

L'application d'une règle de construction identique dans les deux langues ne serait donc ni une interférence, ni un transfert, mais le résultat d'une situation où l'enfant n'a pas encore acquis les contraintes lexicales (Corbin 1987) qui s'appliquent différemment à REdans les deux langues. Alors que la forme phonétique, la position et le sens ont été correctement analysés dans les deux langues, ainsi que la règle de construction, l'enfant en a déduit hâtivement que RE- doit être marqué comme "semblable" et il fait une surgénéralisation de l'application de cette règle au-delà des verbes produisant des énoncés socialement acceptables, conformément à cette hypothèse. Les constructions illégitimes que produit l'enfant bilingue seront parallèles à celles que fait le monolingue sans pour autant qu'il applique un traitement aux deux langues prises comme une seule.

En accord avec Beauvillain (1989:60) qui dit que "l'organisation de la connaissance lexicale chez les bilingues repose sur l'idée selon laquelle la connaissance serait organisée par les «procédures» mises en œuvre lors de son acquisition", il semble que le bilingue 
possède en plus de ce que possèdent deux monolingues un marquage signalant les ressemblances entre les deux langues.

Ce sont les règles générées par un tel marquage qui amèneront l'enfant à faire des surgénéralisations d'une langue à l'autre.

\section{BIBLIOGRAPHIE}

BADECKER, W. et CARAMAZZA, A. 1991. "Morphological composition in the lexical output system". C ognitive Neuropsychology 8 (5), pp. 335 à 367.

Beauvillain, C. 1989. “Traitement lexical et bilinguisme” dans : Lecocq, P. et Segui, J. (eds.) L'accès lexical. Lille : Presses Universitaires de Lille, pp. 52 à 64.

CORBIN, Danielle, (1987), Morphologie dérivationnelle et structuration du lexique, Presses U niversitaires de Lille, 2 VOL.

DE HOUWER, A. “Bilingual language acquisition”. Dans: Fletcher, P. \& McWhinney, B. (eds.), 1995. The Handbook of child language acquisition, Oxford: Blackwell.

GROSJEAN, F. 1982. Life with Two Languages: An Introduction to Bilingualism. Cambridge, MA: Harvard University Press.

HAUGEN, E. 1956. Bilingualism in the Americas: A bibliography and Research Guide. University of Alabama Press.

HOFFMAN, C. 1991. An Introduction to Bilingualism. Essex: Longmans.

KEATLEY, C. et DE GELDER, B. 1992. “The bilingual primed lexical decision task: Cross-language priming disappears with speeded responses”. dans: de Groot, A.M.B. et Barry, C. (eds). 1992. “The Multilingual Community". The European Journal of Cognitive Psychology 4/4, Special Issue.

KLAUSEN, T., SUBRITZKY, M.S. \& HAYASHI, M. 1992. “Initial production of inflections in bilingual childre". Dans: Turner, G. et Messer, D. (eds), à paraître. Critical aspects of language acquisition and development. New York: Macmillan Academic.

LAMBERT, W. 1972. Language, Psychology and Culture. Stanford: Stanford University Press.

QUAY, S. 1998. “The relationship between input and language choice”. Communication présentée au 6ème Congrès International de Pragmatique, Reims, juillet.

VOLTERRA, V. et TAESCHNER, T. 1978. “The acquisition and development of language by bilingual children". Journal of Child Language 5, pp. 311-26.

WEINREICH, U. 1974. Languages in contact: Findings and problems. The Hague : Mouton.

\section{RÉSUMÉS}

Depuis longtemps on a rejeté l'hypothèse que l'enfant acquiert la langue par simple imitation des adultes qui l'entourent et par un conditionnement skinnerien. On considère que l'enfant écoute, 
découpe et analyse le langage qu'il entend et qu'il élabore une structure qui sert de base à ses propres productions. Sa restructuration du langage l'amène parfois à sur-généraliser les règles du langage adulte. On pourrait considérer que le même genre de surgénéralisation se produit chez l'enfant bilingue entre les deux langues, surtout lorsque les langues offrent des ressemblances.

On a remarqué chez un enfant bilingue anglais-français (âge 4,0 à 4,9) une généralisation persistante en anglais du morphème re-, l'amenant à créer des constructions de mots en anglais illégales par rapport à la langue adulte : e.g., *reask, *rego, *recome, *refind, *reput, *recaught. On propose que ces constructions correspondent à une surgénéralisation morphologique faite par l'enfant bilingue dans sa tentative de construire des règles lui permettant d'organiser les différences et les ressemblances entre ses deux langues.

The hypothesis that children learn language through simple imitation of adults has long been discarded. It rather seems that children listen and analyse the language they hear around them, and then synthesize a structure on which they base their own productions. This restructuration of language leads them, at times, to an over-generalisation of the rules of adult language. It would seem that the same type of over-generalisation is sometimes produced across the two languages of a bilingual child, especially when the languages are quite similar.

A great deal of generalisation of the use of the morpheme RE- was found to occur in the English of an English-French bilingual child (age 4,0-4,9), whereby he created a number of constructions not found in adult language : e.g., *reask, *recome, *refind, *reput, *recaught, *rego. The first five of these might be considered a mapping of French onto English, but the last fits neither language. It is suggested here that these constructions represent an over-generalisation of the combinatory rules governing the creation of words with RE- as a prefix, and that their occurrence supports the hypothesis that besides rules governing each of their two languages, bilinguals also set up rules concerning differences and similarities across languages.

\section{AUTEUR}

\section{MARLÈNE DOLITSKY}

IUFM de Paris, mdolitsky@aol.com 